\title{
Accelerated dynamics in adiabatically-tunable asymmetric double-well potential
}

\author{
I. Setiawan ${ }^{1,2,3}$, B.E. Gunara ${ }^{1}$, J. S. Kosasih ${ }^{1}$ and K. Nakamura ${ }^{3,4}$ \\ ${ }^{1}$ Physics Department, Insititut Teknologi Bandung, Jalan Ganesha 10, Bandung 40132, Indonesia \\ ${ }^{2}$ Physics Education Department, University of Bengkulu, Jalan WR, Supratman Kandang Limun, \\ Bengkulu 38371, Indonesia \\ ${ }^{3}$ Faculty of Physics, National University of Uzbekistan, Vuzgorodok, Tashkent 100174, Uzbekistan \\ ${ }^{4}$ Department of Applied Physics, Osaka City University, Sumiyoshi-ku, Osaka 558-8585, Japan \\ isetiawan@unib.ac.id
}

PACS 03.65.Ge, 03.65.Vf

DOI 10.17586/2220-8054-2017-8-1-99-107

\begin{abstract}
We study a scheme for the acceleration of adiabatic quantum dynamics. Masuda-Nakamura's theory of acceleration uses a strategy of combining two opposite ideas: infinitely-large time-magnification factor $(\bar{\alpha})$ and infinitely-small growth rate $(\epsilon)$ for the adiabatic parameter. We apply the proposed method to a system with a parameter-dependent asymmetric double-well potential which has no scale invariance, and obtain the elctromagnetic field required to accelerate the system. The ground state wave function, initially localized in one well, quickly moves to another well. We investigate two kinds of ground-state wave functions: with and without space-dependent phase $\eta$. For the system with non-zero $\eta$, we show the fast-forward of the adiabatic change for current density distribution more clearly characterizes the well-to-well transport and is deformed as the finite renormalized time-multiplication factor $(\bar{v}=\epsilon \bar{\alpha})$ is varied.
\end{abstract}

Keywords: fast-forwarding, adiabatic quantum dynamics, asymmetric double-well potential.

Received: 14 July 2016

Revised: 2 September 2016

\section{Introduction}

The shorter production time in manufacturing products (e.g., electronics, automotives, plants, etc.) is becoming an important factor in nanotechnology. Currently, we can control even individual atoms [1] and macroscopic wave packets in BEC [2-5]. If we try to fabricate massive amounts of such nanoscale stuctures, we should shorten the dynamics of each atom or BEC to get its desired target states in shorter times. A theory to accelerate quantum dynamics is proposed by Masuda and Nakamura [6] with use of additional phase and driving potential. This theory aims to accelerate a known quantum evolution and to obtain the desired target states on shorter time scales, by fast forwarding the standard quantum dynamics. The theory of fast-forward can be developed to accelerate the adiabatic quantum dynamics [7], and constitutes one of the most promising means to obtain a shortcut to adiabaticity [8-14]. The relationship between the acceleration and the shortcut to adiabaticity is presently clear $[15,16]$. Adiabaticity occurs when the external parameter of Hamiltonian is very-slowly changed. The quantum adiabatic theorem [17-21] states that if the system is initially in an eigenstate of the instantaneous Hamiltonian, it remains so during the adiabatic process. In section 2, we briefly summarize the theory of fastforward of quantum adiabatic dynamics $[6,7]$.

\section{The theory of fast-forward by Masuda and Nakamura}

Let $\Psi_{0}(x, t)$ be a known function of space $(x)$ and time $(t)$. We shall call it a standard state. Let $\alpha$ the time magnification factor of the acceleration. Time evolution of the wave funtion (WF) is sped up if $\alpha>1$ and slowed down if $0<\alpha<1$. In general $\alpha$ can be time dependent $\alpha=\alpha(t)$. The time evolution of the WF is accelerated and decelerated when $\alpha(t)$ is increases and decreases, respectively. The ideal accelerated state is defined as:

$$
\left|\Psi_{\alpha}(t)\right\rangle \equiv\left|\Psi_{0}(\Lambda(t))\right\rangle
$$

where

$$
\Lambda(t)=\int_{0}^{t} \alpha\left(t^{\prime}\right) d t^{\prime},
$$

but it proves difficult to realize $\left|\Psi_{\alpha}(t)\right\rangle$. Consequently, Nakamura and Masuda redefined the accelerated state with use of an additional phase factor $f(x, t)$ as:

$$
\left|\Psi_{\mathrm{FF}}(x, t)\right\rangle=\exp [i f(x, t)]\left|\Psi_{a}(x, t)\right\rangle .
$$


The dynamics for the standard state is assumed to be described by Schrödinger equation:

$$
i \hbar \frac{d \Psi_{0}}{d t}=-\frac{\hbar^{2}}{2 m_{0}} \nabla^{2} \Psi_{0}+V_{0}(x, t) \Psi_{0} .
$$

The corresponding equation for the accelerated state is:

$$
i \hbar \frac{d \Psi_{\mathrm{FF}}}{d t}=-\frac{\hbar^{2}}{2 m_{0}} \nabla^{2} \Psi_{\mathrm{FF}}+V_{\mathrm{FF}} \Psi_{\mathrm{FF}}
$$

where $V_{\mathrm{FF}}$ is the driving potential.

By using Eqs. (1)-(5), the expression for $f$ and $V_{\mathrm{FF}}$ can be written, respectively, as:

$$
f(x, t)=(\alpha(t)-1) \eta(x, \Lambda(t))
$$

and

$$
V_{\mathrm{FF}}=V_{0}-\hbar \eta \frac{d \alpha}{d t}-\hbar \frac{\alpha^{2}-1}{\alpha} \frac{\partial \eta}{\partial t}-\frac{\hbar^{2}}{2 m_{0}}\left(\alpha^{2}-1\right)(\nabla \eta)^{2},
$$

where $\eta \equiv \eta(x, \Lambda(t))$ is the phase of the standard state $\Psi_{0}$.

Next, we study the fast-forwarding of adiabatic quantum dynamics. Masuda and Nakamura [7] proposed a theory to accelerate the adiabatic quantum dynamics by using a combination of opposite ideas of infinitely-large time multiplication and infinitesimally-slow evolution rate of adiabatic dynamics, to generate the finite driving potential, by which we obtain the final adiabatic state in any desired short time. To apply the theory of accelerated standard dynamics, it is necessary to regularize the adiabatic state so that it satisfies the time-dependent Schrödinger equation. A regularized potential and $\mathrm{WF}$ is written a

$$
\begin{gathered}
V_{0}^{\mathrm{reg}}=V_{0}+\epsilon \tilde{V}(x, t), \\
\Psi_{0}^{\mathrm{reg}}(x, t, R(t))=\phi_{n}(x, R(t)) e^{(-i / \hbar) \int_{0}^{t} E_{n}\left(R\left(t^{\prime}\right)\right) d t^{\prime}} e^{i \epsilon \theta(x, t)},
\end{gathered}
$$

where $R$ is a time-dependent adiabatic parameter as:

$$
R(t)=R(0)+\epsilon t
$$

with $\epsilon \ll 1$, and: $\phi_{n}$ is the eigenstate of the time-independent Schrödinger equation,

$$
H \phi_{n}=E_{n} \phi_{n} \text {. }
$$

Formally, $\phi_{n}$ can be decomposed as:

$$
\phi_{n}=\bar{\phi}_{n} e^{i \eta(x, t)},
$$

where $\bar{\phi}_{n}$ is a real positive amplitude and $\eta$ is the phase. The Schrödinger equation for $\Psi_{0}^{\text {reg }}$ is represented as:

$$
i \hbar \frac{d \Psi^{\mathrm{reg}}}{d t}=-\frac{\hbar^{2}}{2 m_{0}} \nabla^{2} \Psi^{\mathrm{reg}}+V_{0}^{\mathrm{reg}}(x, t) \Psi^{\mathrm{reg}} .
$$

$\Psi_{0}^{\text {reg }}$ and $V_{0}^{\text {reg }}$ are assumed to satisfy Eq. (13) up to $O(\epsilon)$. By subtituting Eqs. (8), (9) and (12) into Eq. (13) and decomposing it into real and imaginary parts, we have $\theta$ and $\tilde{V}$ as follows:

$$
\bar{\phi}_{n}^{2} \nabla^{2} \theta+2 \bar{\phi}_{n} \nabla \bar{\phi}_{n} \nabla \theta+\frac{2 m_{0}}{\hbar} \bar{\phi}_{n} \frac{\partial \bar{\phi}_{n}}{\partial R}=0,
$$

and

Here, $\theta$ can be solved as:

$$
\tilde{V}=-\hbar\left(\partial_{R} \eta\right)-\frac{\hbar^{2}}{m_{0}} \nabla \eta \nabla \theta .
$$

$$
\nabla \theta=-\frac{m_{0}}{\hbar} \frac{1}{\bar{\phi}_{n}^{2}(x, R)} \frac{\partial}{\partial R} \int_{-\infty}^{x} \bar{\phi}_{n}^{2}\left(x^{\prime}, R\right) d x^{\prime} .
$$

Applying the general issue in Eqs. (6) and (7), we have:

$$
f(x, t)=(\alpha(t)-1) \epsilon \theta(\mathrm{x}, R(\Lambda(t)) .
$$

With use of $f$ in Eq. (17), the driving potential $V_{\mathrm{FF}}$ to realize fast-forward of adiabatic quantum dynamics can be written as:

$$
\begin{aligned}
& V_{\mathrm{FF}}=-\alpha \epsilon \hbar\left(\partial_{R} \eta\right)-\alpha \epsilon \frac{\hbar^{2}}{m_{0}} \nabla \eta \nabla \theta \\
& -\hbar \frac{d \alpha}{d t} \epsilon \theta-\hbar \epsilon^{2} \alpha^{2} \frac{\partial \theta}{\partial R}-\frac{\hbar^{2}}{2 m_{0}} \epsilon^{2} \alpha^{2}(\nabla \theta)^{2}+V_{0} .
\end{aligned}
$$


The expression for driving electric field can be written as:

$$
\begin{aligned}
& \hat{E}_{\mathrm{FF}} \equiv-\frac{\partial}{\partial x}\left(V_{\mathrm{FF}}-V_{0}\right)=\hbar \epsilon \dot{\alpha} \nabla \theta+\hbar \epsilon^{2} \alpha^{2} \frac{\partial}{\partial R}(\nabla \theta)+\frac{\hbar^{2}}{m_{0}}(\alpha \epsilon)^{2} \nabla \theta \nabla^{2} \theta \\
& +\alpha \epsilon \hbar \partial_{R}(\nabla \eta)+\alpha \epsilon \frac{\hbar^{2}}{m_{0}} \nabla(\nabla \eta \nabla \theta) .
\end{aligned}
$$

Taking the limit $\epsilon \rightarrow 0, \bar{\alpha} \rightarrow \infty$, with $\epsilon \bar{\alpha}=\bar{v}$, we can define $v(t)$ and $\dot{v}(t)$ as:

$$
\begin{gathered}
\alpha \epsilon=\bar{v}\left(1-\cos \frac{2 \pi t}{T_{F}}\right) \equiv v(t), \\
\dot{\alpha} \epsilon=\dot{v}(t) .
\end{gathered}
$$

Then, $\Psi_{\mathrm{FF}}$ becomes $\Psi_{\mathrm{FF}}=\bar{\phi}_{n} e^{i(\eta+v(t) \theta)}$, and $\hat{E}_{\mathrm{FF}}$ is given by:

$$
\begin{aligned}
& \hat{E}_{\mathrm{FF}}=\hbar \dot{v}(t) \nabla \theta+\hbar(v(t))^{2} \partial_{R} \nabla \theta+\frac{\hbar^{2}}{m_{0}}(v(t))^{2} \nabla \theta \nabla^{2} \theta \\
& +v(t) \hbar \partial_{R}(\nabla \eta)+v(t) \frac{\hbar^{2}}{m_{0}} \nabla(\nabla \eta \nabla \theta) .
\end{aligned}
$$

The magnification factor $\alpha$ in Eq. (20) is commonly chosen for $0 \leq t \leq T_{F}$, where $\bar{v}$ is the time average of $\alpha(t) \epsilon$ during the fast forwarding. The final time of the fast-forward $T_{F}$ is related to the standard final time $T$ as $T_{F}=\epsilon T / \bar{v}$, where $T$ is taken as $T=1 / \epsilon(\gg 1)$ and $R(T)-R(0)=1$.

The driving electric field $\left(\hat{E}_{\mathrm{FF}}\right)$ guarantees the fast-forward of adiabatic quantum dynamics [22]. Recently, Nakamura et al. [23] suggested a new theory for the fast-forwarding of the adiabatic dynamics without the additional phase $f$. But the present and new theories are compatible by using the gauge transformation as shown in Appendix A.

\section{Accelerated dynamics in adiabatically tunable-asymmetric double-well potential: case of $\tilde{V}=0$}

We show the acceleration of the adiabatic quantum dynamics with use of a parameter-dependent asymmetric double-well potential, which does not satisfy the scale invariance such as dilation or translation invariance. Let's define the zero energy $\left(E_{0}=0\right)$ eigen-function which is a superposition of a pair of Gaussian functions with their center at $x=0$ and $x=d$, where $d$ represents the distance between the two wells. To be explicit:

$$
\Psi_{0}=h(R)\left[(1-R) e^{-\frac{x^{2}}{2 a}}+R e^{-\frac{(x-d)^{2}}{2 a}}\right],
$$

changing in the range $0 \leq R \leq 1$. The potential $V_{0}(x)$ to realize $\Psi_{0}$ is written by:

$$
V_{0}(x) \equiv \frac{\hbar^{2}}{2 m} \frac{\Psi_{0}^{\prime \prime}}{\Psi_{0}}=\frac{\hbar^{2}}{2 m_{0}}\left[-\frac{1-R}{a}\left(1-\frac{x^{2}}{a}\right)-\frac{R}{a}\left(1-\frac{(x-d)^{2}}{a}\right) f(x)\right] \times \frac{1}{(1-R)+R f(x)},
$$

with $f(x)=e^{\frac{d}{a}\left(x-\frac{d}{2}\right)}$. The normalization factor $h(R)$, determined by $\int_{-\infty}^{\infty}|\Psi|^{2}=1$, is given by:

$$
h(R)=\frac{1}{(\pi a)^{1 / 4}}\left(1-2 R+2 R^{2}+2 R(1-R) e^{-\frac{d^{2}}{4 a}}\right)^{-1 / 2} .
$$

Figures 1 and 2 show the change of the potential and wave function for various values of $R$, between $R=0$ and $R=1$. As the potential minimum moves from $x=0$ to $x=d$, the center-of-mass of WF also moves in the same direction. Fig. 3 shows the probability amplitude $\left|\Psi_{0}\right|^{2}$ in the adiabatic dynamics with $R(t)=\epsilon t$. Fig. 4 shows $\left|\Psi_{\mathrm{FF}}\right|^{2}$ in the fast-forwarded adiabatic dynamics, where $R(\Lambda(t))$ is written as:

$$
R(\Lambda(t))=\epsilon \int_{0}^{t} \alpha\left(t^{\prime}\right) d t^{\prime}=\int_{0}^{t} v\left(t^{\prime}\right) d t^{\prime}=\bar{v}\left(t-\frac{T_{F}}{2 \pi} \sin \frac{2 \pi t}{T_{F}}\right)
$$

The adiabatic standard dynamics evolves up to the final time $T=10^{2}$ with use of $\epsilon=10^{-2}$ and its accelerated version up to the shortened final time $T_{\mathrm{F}}=T / \bar{\alpha}=\epsilon T / \bar{v}=0.025$ with use of the mean acceleration factor $\bar{\alpha}=4 \times 10^{3}$ and mean velocity $\bar{v}=\bar{\alpha} \epsilon=40$. 


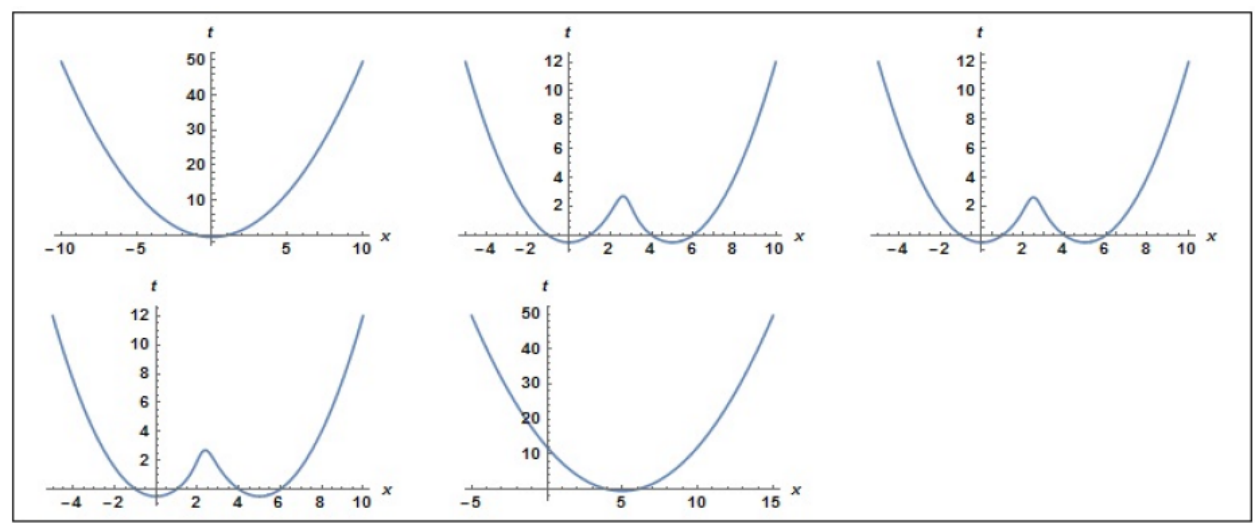

FIG. 1. The change of $V_{0}(x)$ with various values of $R: R=0 \rightarrow 0.25 \rightarrow 0.5 \rightarrow 0.75 \rightarrow 1$ (from top left to bottom right), $d=5$

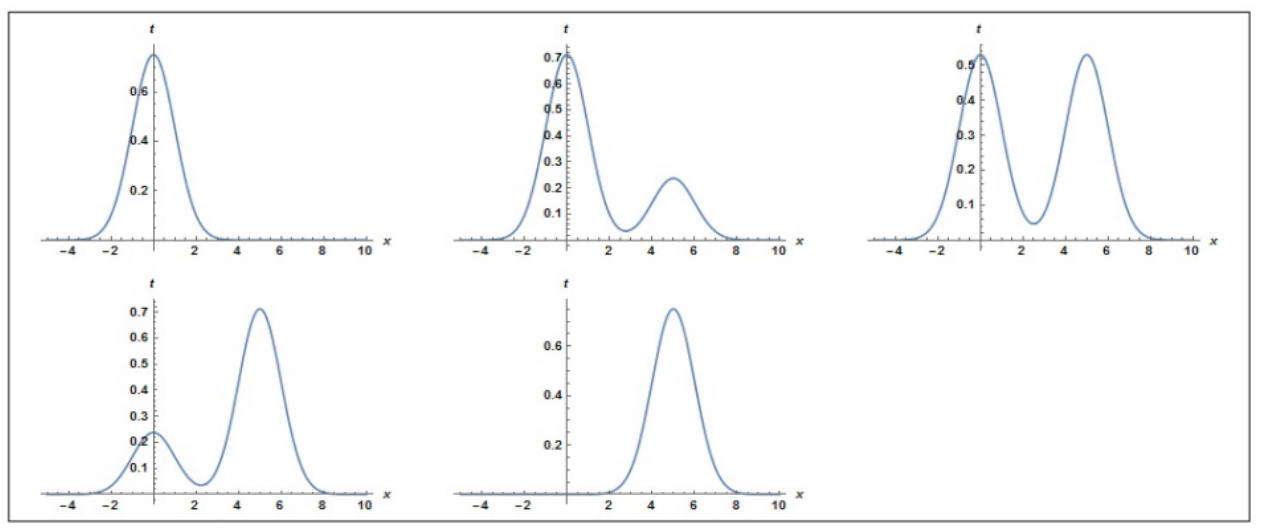

FIG. 2. The change of probability amplitude $\left|\Psi_{0}(x)\right|$ with various values of $R: R=0 \rightarrow 0.25 \rightarrow$ $0.5 \rightarrow 0.75 \rightarrow 1, d=5$

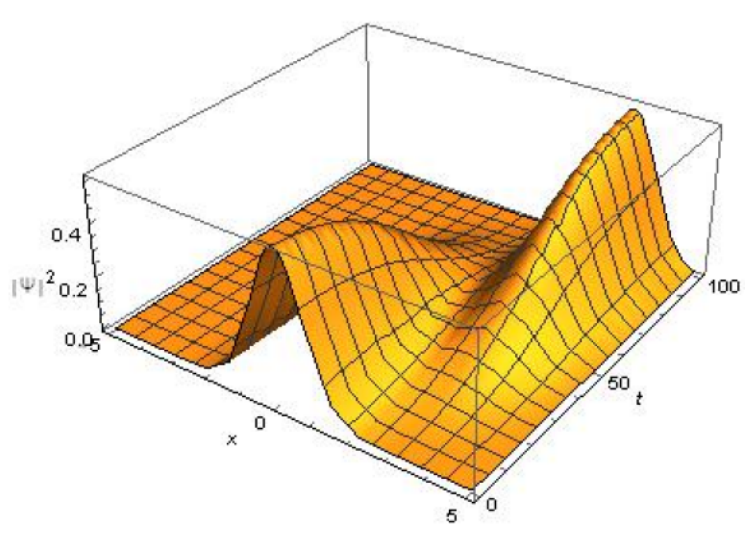

FIG. 3. 3D plot of $|\hat{\Psi}|^{2}$ with $\epsilon=10^{-2}, T=100, a=1$, and $d=3$

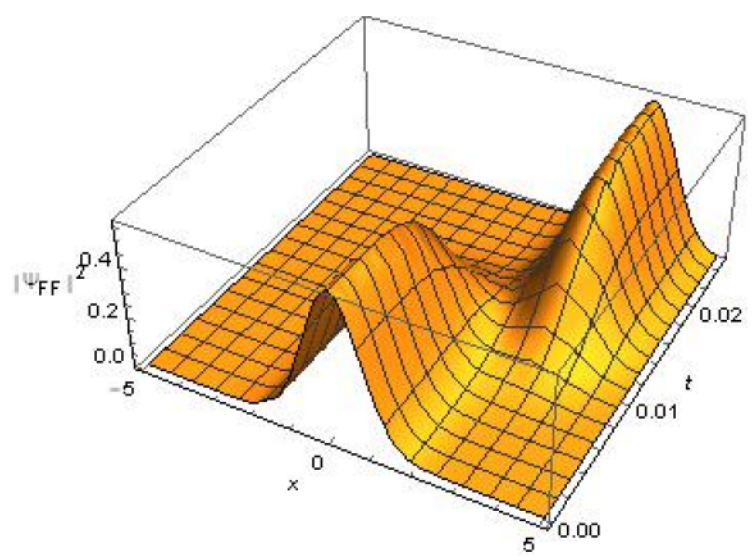

FIG. 4. 3D plot of $\left|\hat{\Psi}_{\mathrm{FF}}\right|^{2}$ with $T_{F}=T / \bar{\alpha}=0.025$

From Eqs. (16), $\nabla \theta$ is given by:

$$
\nabla \theta=-\frac{1}{2}(\pi a)^{1 / 2}\left[\frac{\kappa}{\left((1-R) e^{-\frac{x^{2}}{2 a}}+R e^{-\frac{(x-d)^{2}}{2 a}}\right)^{2}}-\frac{\left(-2+4 R+(2-4 R) e^{-\frac{d^{2}}{4 a}}\right) \mu}{\left((1-R) e^{-\frac{x^{2}}{2 a}}+R e^{-\frac{(x-d)^{2}}{2 a}}\right)^{2} g}\right],
$$


with

and

$$
\begin{gathered}
\kappa=(2-4 R) e^{-\frac{d^{2}}{a}} \operatorname{erfc}\left(-\frac{x-\frac{d}{2}}{\sqrt{a}}\right)+2 R \operatorname{erfc}\left(-\frac{x-d}{\sqrt{a}}\right)+(2 R-2) \operatorname{erfc}\left(-\frac{x}{\sqrt{a}}\right), \\
\mu=\left(2 R(1-R) e^{-\frac{d^{2}}{a}} \operatorname{erfc}\left(-\frac{x-\frac{d}{2}}{\sqrt{a}}\right)+R^{2} \operatorname{erfc}\left(-\frac{x-d}{\sqrt{a}}\right)+(1-R)^{2} \operatorname{erfc}\left(-\frac{x}{\sqrt{a}}\right)\right),
\end{gathered}
$$

where the complimentary error function erfc is :

$$
g=\left(2(1-R) R e^{-\frac{d^{2}}{4 a}}+2 R^{2}-2 R+1\right)
$$

$$
\operatorname{erfc}(x)=\frac{2}{\sqrt{\pi}} \int_{x}^{\infty} e^{-t^{2}} d t
$$

The driving electric field $\left(\hat{E}_{\mathrm{FF}}\right)$ to generate the acceleration is calculated by Eq. (22) and is shown in Fig. 5.

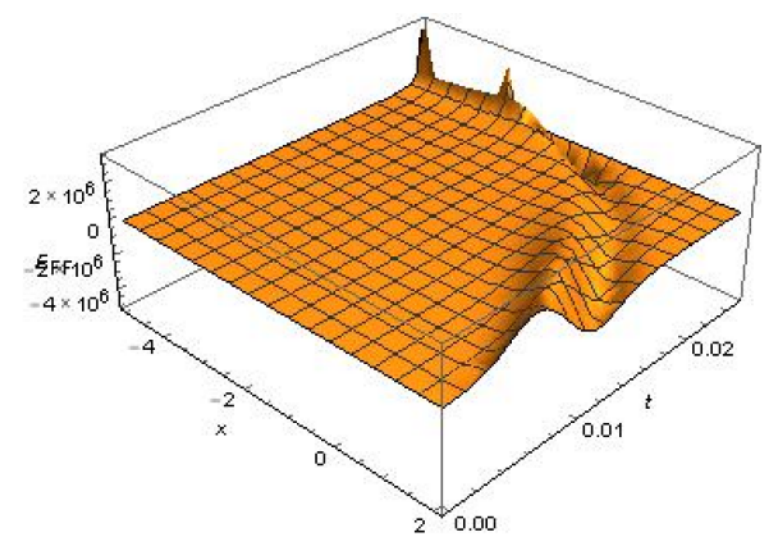

FIG. 5. $\hat{E}_{\mathrm{FF}}$ with $T_{F}=0.025$

\section{The case of $\tilde{V} \neq 0$}

In the previous example, the right-hand side of Eq. (15) was always vanishing, because of the absence of a space-dependent phase $\eta$ in the standard WF. In many practical cases, we have an adiabatic state with a spacedependent phase $\eta$. Choosing again the asymmetric double-well potential, we shall investigate the fast-forwarding with non-zero $\tilde{V}$ by having recourse to a stationary state with a phase $\eta$. Here, the adiabatic state with the eigenvalue $E_{n=0}=0$ is chosen as:

$$
\Psi_{0}=h(R)\left[(1-R) e^{-\frac{x^{2}}{2 a}}+R e^{-\frac{(x-d)^{2}}{2 a}}\right] e^{i \eta},
$$

with $\eta$ given as:

$$
\eta=B \int_{-\infty}^{x} \exp \left[-\frac{1}{h(R)\left((1-R) e^{-\frac{x^{2}}{2 a}}+R e^{-\frac{(x-d)^{2}}{2 a}}\right)^{2}}\right],
$$

where $B$ is a real constant. The potential that guarantees the eigenstate in Eq. (29) is written as:

$$
\begin{aligned}
& V_{0}(x)=\frac{\hbar^{2}}{2 m_{0}}\left[\left(-\frac{1-R}{a}\left(1-\frac{x^{2}}{a}\right)-\frac{R}{a}\left(1-\frac{(x-d)^{2}}{a}\right) f(x)\right)\right. \\
& \left.\times \frac{1}{(1-R)+R f(x)}+\frac{1}{2} \exp \left(-\frac{1}{h(R)\left((1-R) e^{-\frac{x^{2}}{2 a}}+R e^{-\frac{(x-d)^{2}}{2 a}}\right)^{2}}\right)\right],
\end{aligned}
$$

with $f(x)=e^{\frac{d}{a}\left(x-\frac{d}{2}\right)}$, and $h(R)$ being given in Eq. (25). Eq. (31) is available from Eq. (24) by adding an extra term. $\nabla \theta$, which appears in the regularization procedure, is the same as in Eq. (27) because of the formula Eq. (16). From Eqs. (15) and (29), $\tilde{V}$ is written as: 


$$
\begin{aligned}
& \tilde{V}=-\hbar \frac{\partial}{\partial_{R}}\left(B \int_{-\infty}^{x} \exp \left[-\frac{1}{h(R)\left((1-R) e^{-\frac{x^{2}}{2 a}}+R e^{\left.-\frac{(x-d)^{2}}{2 a}\right)^{2}}\right.}\right]\right) \\
& -\frac{\hbar^{2}}{m_{0}} B \exp \left[-\frac{1}{h(R)\left((1-R) e^{-\frac{x^{2}}{2 a}}+R e^{-\frac{(x-d)^{2}}{2 a}}\right)^{2}}\right] \nabla \theta .
\end{aligned}
$$

Figures 6 and 7 show the amplitude of the standard wave function and its fast-forward version with $\eta \neq 0$, respectively. $\hat{E}_{\mathrm{FF}}$ is evaluated by Eqs. (22), and is depicted in Fig. 8. We see no big difference of fast forward between the cases with $\eta=0$ and $\eta \neq 0$.

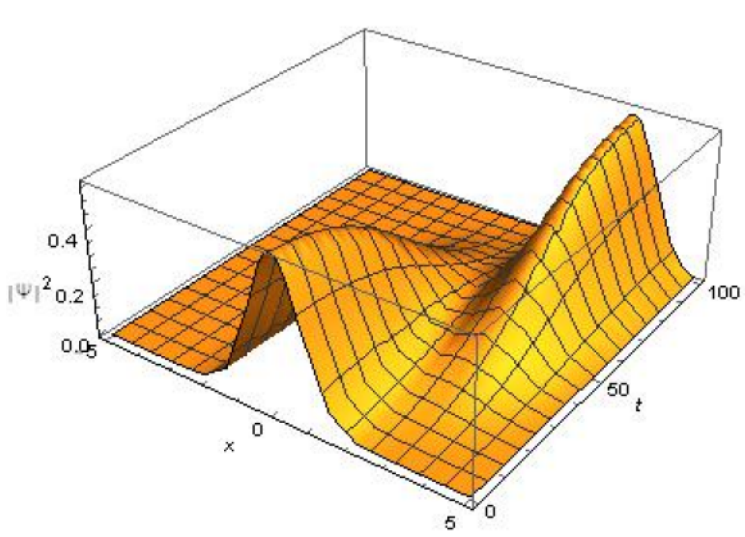

FIG. 6. 3D plot of $|\hat{\Psi}|^{2}$ with $\eta \neq 0$ and $B=1$

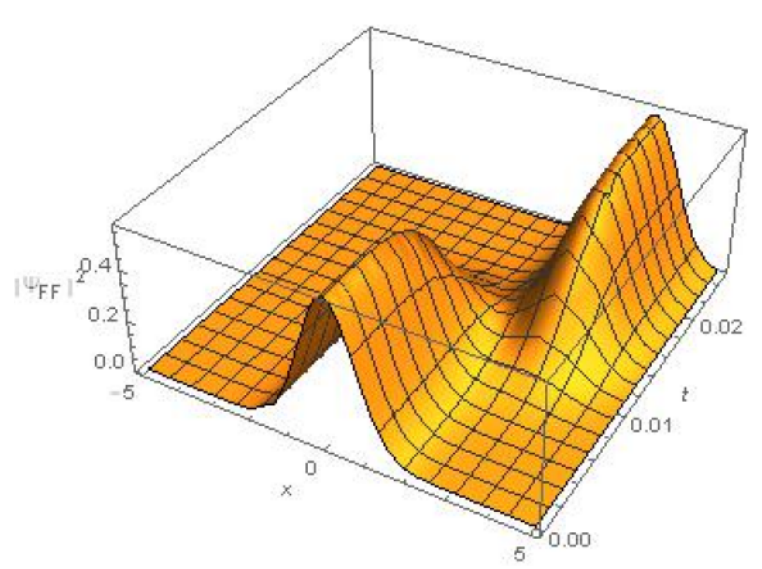

FIG. 7. 3D plot of $\left|\hat{\Psi}_{\mathrm{FF}}\right|^{2}$ with $\eta \neq 0$, $T_{F}=T / \bar{\alpha}=0.025$ and $B=1$

The present scheme also introduces a current density $j_{\mathrm{FF}}$. We see:

and find the relationship:

$$
\begin{gathered}
j_{\mathrm{FF}}(x, t)=\operatorname{Re}\left[\Psi_{\mathrm{FF}} \frac{\hbar}{i m} \nabla \Psi_{\mathrm{FF}}\right]= \\
j(x, t)=\frac{\hbar}{m} \bar{\phi}_{n}^{2}(x, \Lambda(t))[\nabla \eta(x, \Lambda(t))+v(t) \nabla \theta(x, \Lambda(t))], \\
\bar{\phi}_{n}^{2}(x, t) \nabla \eta(x, t),
\end{gathered}
$$

$$
j_{\mathrm{FF}}(x, t)=j(x, \Lambda(t))+\frac{\hbar}{m} v(t) \bar{\phi}_{n}^{2}(x, \Lambda(t)) \nabla \theta(x, \Lambda(t)) .
$$

Thus, $j_{\mathrm{FF}}$ has a non-adiabatic current in addition to the adiabatic one $j(x, \Lambda(t))$. Figs. 9 and 10 show the adiabatic current density and fast-forward current density respectively. We find : the adiabatic current density distribution in Fig. 9 is radically changed by the fast forwarding. This change, in particular, the increase of the current intensity becomes more marked as the control parameter $\bar{v}$ associated with the fast forwarding is increased (see Fig. 10). At the end of the fast forwarding, the non-adiabatic current vanishes.

The asymmetric double-well potential in the present paper breaks the scale invariance. In the case of a scaleinvariant single well, Lewis-Riesenfeld's invariant method [11] can also lead to the accelerated dynamics, which will be discussed in Appendix B.

\section{Conclusion}

The Masuda-Nakamura's scheme of accelerated adiabatic quantum dynamics is applied to the system with a tunable asymmetric double-well potential and the driving electric field is obtained. The potential here has no scale invariance. A combination of opposite ideas of infinitely-large time multiplication and infinitesimally-slow adiabatic dynamics generates a finite driving potential, by which we obtain the final adiabatic state in any desired short time. We investigated two kinds of ground state wave functions, with and without the space-dependent phase $\eta$. For the system with non-zero $\eta$, we see that the fast-forward of current density acquires a non-adiabatic 


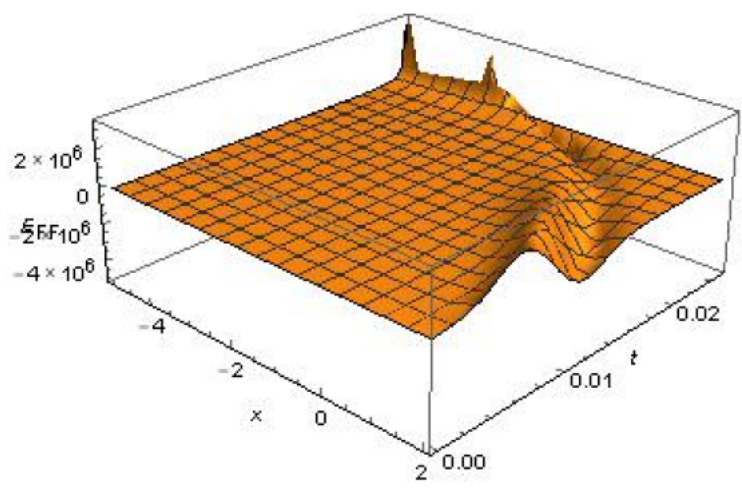

FIG. 8. $\hat{E}_{\mathrm{FF}}$ with $\eta \neq 0, T_{F}=T / \bar{\alpha}=$ 0.025 and $B=1$

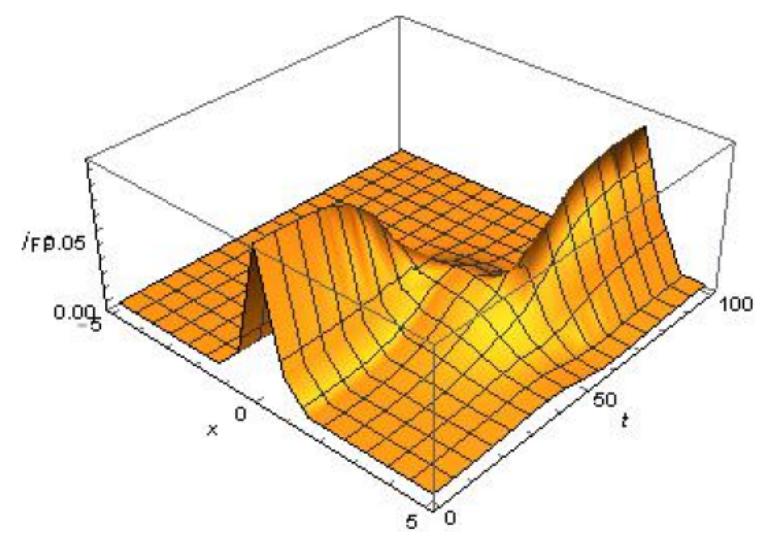

(a)

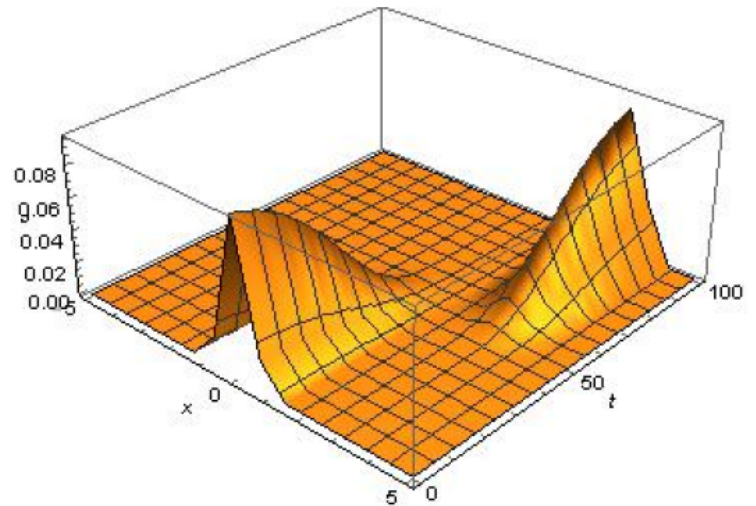

FIG. 9. 3D plot of $j$ with $B=1$

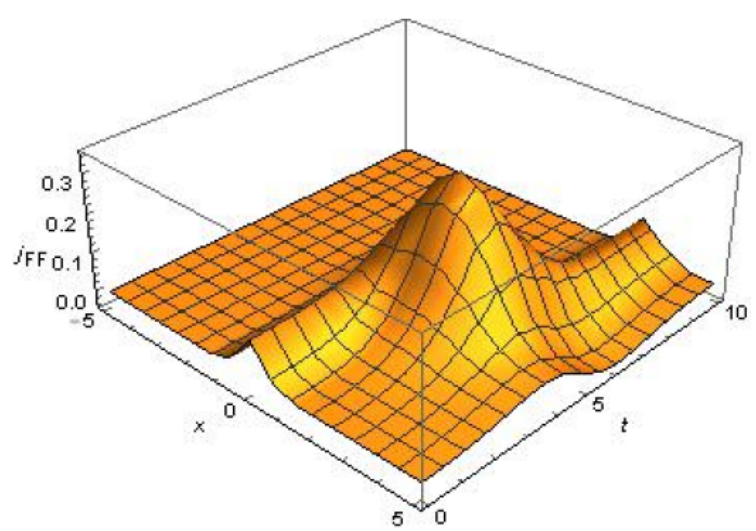

(b)

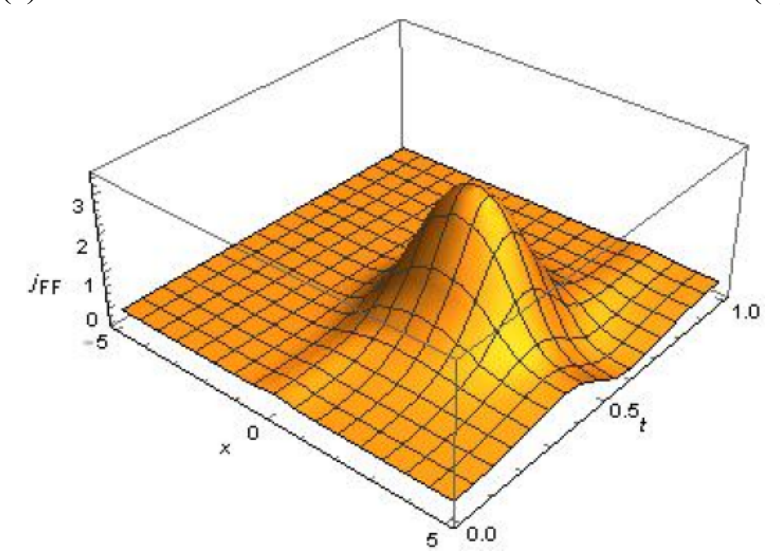

(c)

FIG. 10. 3D plot of $j_{\mathrm{FF}}$ with $\eta \neq 0$ and $B=1$ : (a) $\bar{\alpha}=1, \bar{v}=0.01, T_{\mathrm{F}}=100$; (b) $\bar{\alpha}=10$, $\bar{v}=0.1, T_{\mathrm{F}}=10 ;$ (c) $\bar{\alpha}=10^{2}, \bar{v}=1, T_{\mathrm{F}}=1$

contribution besides the adiabatic current density and that the current intensity radically grows as the control parameter associated with fast forwarding is increased.

\section{Acknowledgements}

This work is funded by Riset KK ITB 2015-2016 and Hibah Desentralisasi ITB 2015-2016. I. S. is grateful to Prof. Davron Matrasulov for his great hospitality at Turin Polytechnical University at Tashkent. 


\section{Appendix A. Gauge tranformation to the scheme without the additional phase}

Let's consider the gauge transformation as follows :

with

$$
\begin{aligned}
\Psi_{\mathrm{FF}} & \rightarrow \hat{\Psi}_{\mathrm{FF}} e^{i \zeta} \\
V_{\mathrm{FF}} & \rightarrow \hat{V}_{\mathrm{FF}}-\frac{\hbar}{q} \dot{\zeta} \\
A_{\mathrm{FF}}(=0) & \rightarrow \hat{A}_{\mathrm{FF}}+\frac{\hbar c}{q} \nabla \zeta,
\end{aligned}
$$

$$
\zeta=\epsilon \alpha(t) \theta(x, R(\Lambda(t)))
$$

Then, the Schrödinger equation becomes that of a charge particle under the gauge potentials $\hat{A}_{\mathrm{FF}}$ and $\hat{V}_{\mathrm{FF}}$ :

$$
i \hbar \frac{\partial \hat{\Psi}_{\mathrm{FF}}}{\partial t}=\frac{1}{2 m_{0}}\left(\frac{\hbar}{i} \nabla-\frac{q}{c} \hat{A}_{\mathrm{FF}}\right)^{2} \hat{\Psi}_{\mathrm{FF}}+q \hat{V}_{\mathrm{FF}} \hat{\Psi}_{\mathrm{FF}} .
$$

The wave function becomes free from the additional phase :

$$
\hat{\Psi}_{\mathrm{FF}}=\phi_{n}(x, R(\Lambda(t))),
$$

and the scalar and vector potential are given by:

$$
\begin{gathered}
\hat{V}_{\mathrm{FF}}=-\frac{\hbar^{2}}{2 m_{0}} \epsilon^{2} \alpha^{2}(\nabla \theta)^{2}-\alpha \epsilon \hbar\left(\partial_{R} \eta\right)-\alpha \epsilon \frac{\hbar^{2}}{m_{0}} \nabla \eta \nabla \theta, \\
\hat{A}_{\mathrm{FF}}=-\hbar \epsilon \alpha \nabla \theta
\end{gathered}
$$

respectively. Equations (A.2)-(A.5) are nothing but the scheme proposed in Ref. [23].

\section{Appendix B. Relationship between Masuda-Nakamura's scheme and Lewis-Riesenfeld method}

Let us consider the Hamiltonian for single well potential:

$$
H=\frac{\hat{p}^{2}}{2 m}+\frac{1}{2} m \omega^{2}(t) \hat{x}^{2},
$$

where $\omega$ is a time-dependent parameter. The wave function at any time $t$ can be deduced from Lewis-Riesenfeld theory of invariants [11]. By assuming the existence of a Hermitian invariant of the homogeneous, its quadratic form is given by:

$$
I(t)=\frac{1}{2}\left[\alpha(t) \hat{x}^{2}+\beta(t) \hat{p}^{2}+\gamma(t)(\hat{x} \hat{p}+\hat{p} \hat{x})\right],
$$

where $\alpha, \beta$, and $\gamma$ are real function of time. $I(t)$ should satisfy the conditions:

$$
\frac{d I}{d t}=\frac{\partial I}{\partial t}+\frac{1}{i \hbar}[I, H]=0
$$

In order to satisfy Eq. (B.3), it is convenient to introduce another function $\sigma(t)$, defined by:

$$
\beta(t)=\sigma^{2}(t),
$$

where $\sigma^{2}(t)$ is a real function of time. By making the scale transformation $\sigma(t)=c^{1 / 4} \rho(t)$, the invariant may be written in the form:

$$
I=\frac{1}{2} m \omega_{0}^{2}\left(1 / \rho^{2}\right) \hat{x}^{2}+\frac{1}{2 m}(\rho \hat{p}-m \dot{\rho} \hat{x})^{2} .
$$

Here, $c$ is an arbitrary constant of integration and $\rho$ satisfies the Ermakov equation:

$$
\ddot{\rho}+\omega^{2}(t) \rho-\frac{\omega_{0}^{2}}{\rho^{3}}=0 .
$$

The eigenstates and the eigenvalues of the invariant operator $I(t)$ may be found by an operator technique. Thus the dynamical phase function may be written in the form:

$$
\alpha_{n}(t)=-\frac{i}{\hbar}\left(n+\frac{1}{2}\right) \int_{0}^{t} d t^{\prime} \frac{1}{\rho^{2}\left(t^{\prime}\right)} .
$$

For the Hamiltonian in Eq. (B.1), the general solution of time-dependent Schrödinger equation can be expanded as a linear combination with eigenvectors $\psi_{n}$ of $I$ as:

$$
\psi(x, t)=\Sigma_{n} c_{n} e^{i \alpha_{n}} \psi_{n}(x, t)=\Sigma_{n} c_{n} \psi_{n}(x, t) e^{-\frac{i}{\hbar}\left(n+\frac{1}{2}\right) \int_{0}^{t} d t^{\prime} \rho^{-2}\left(t^{\prime}\right)} .
$$


Formally $\psi_{n}$ can be decomposed as $\psi_{n}=\bar{\psi}_{n} e^{i \zeta}$, where $\zeta=\frac{m}{2 \hbar} \frac{\dot{\rho}}{\rho} x^{2}$.

By using $\omega$ in Eq. (B.6), the potential for the single well in Eq. (B.1) can be written as:

$$
V(x, t)=\frac{1}{2} m\left[\frac{\omega_{0}^{2}}{\rho^{4}(t)}-\frac{\ddot{\rho}}{\rho(t)}\right] x^{2} .
$$

The corresponding $V_{0}$ is:

$$
V_{0}(x, t)=\frac{1}{2} m \frac{\omega_{0}^{2}}{\rho^{4}(t)} x^{2}
$$

Applying Eq. (16) to the adiabatic eigenstate of Eq. (B.1), we can show the equality:

$$
\zeta=\epsilon \alpha(t) \theta\left(x, R(\Lambda(t))=\frac{m}{2 \hbar} \frac{\dot{\rho}}{\rho} x^{2}\right.
$$

where $\Lambda(t)=\int_{0}^{t} \alpha\left(t^{\prime}\right) d t^{\prime}$. By making space and time derivatives of Eq. (B.11), the potential in Eq. (B.9) can be written as:

$$
V(x, t)=V_{0}-\hbar \frac{d \alpha}{d t} \epsilon \theta-\hbar \epsilon^{2} \alpha^{2} \frac{\partial \theta}{\partial R}-\frac{\hbar^{2}}{2 m} \epsilon^{2} \alpha^{2}(\nabla \theta)^{2},
$$

which is nothing but $V_{\mathrm{FF}}$ in Eq. (18) in the case $\eta=0$. Therefore, Lewis-Riesenfeld scheme is equivalent to Masuda-Nakamura's. In contrast with [12] and [15], $\alpha(t)$ is an arbitrary and experimentally-accessible time scaling factor and is not necessary to satisfy the Ermakov equation with 6 boundary conditions. Besides this advantage, the Masuda-Nakamura's scheme is applicable to any kind of scale-noninvariant potentials.

\section{References}

[1] Eigler D.E., Schweizer E.K. Positioning single atoms with a scanning tunnelling microscope. Nature (London), 1994, 344, P. 524.

[2] Legget A.J., Schweizer E.K. Bose-Einstein condensation in the alkali gases: Some fundamental concepts. Rev. Mod. Phys., 2001, 73, P. 307.

[3] Ketterle W. Nobel lecture: When atoms behave as waves: Bose-Einstein condensation and the atom laser. Rev. Mod. Phys., 2002, 74, P. 1131.

[4] Learnhardt A.E., Chikkatur A.P., et al. Propagation of Bose-Einstein Condensates in a Magnetic Waveguide. Phys. Rev. Lett., 2002, 89, P. 040401.

[5] Gustavson T.L., Chikkatur A.P., et al. Transport of Bose-Einstein Condensates with Optical Tweezers. Phys. Rev. Lett., 2001, 88, P. 020401.

[6] Masuda S., Nakamura K. Fast-forward problem in quantum mechanics. Phys. Rev. A, 2008, 78, P. 062108.

[7] Masuda S., Nakamura K. Fast-forward of adiabatic dynamics in quantum mechanics. Proc. R. Soc. A, 2010, 466, P. 1135-1154.

[8] Demirplak M., Rice S.A. Adiabatic population transfer with control fields. J. Phys. Chem. A, 2003, 89, P. 9937.

[9] Demirplak M., Rice S.A. Assisted adiabatic passage revisited. J. Phys. Chem. B, 2005, 109, P. 6838.

[10] Berry M.V. Transitionless quantum driving. J. Phys A: Math. Theor., 2009, 42, P. 365303.

[11] Lewis H.R., Riesenfeld W.B. An exact quantum theory of the time-dependent harmonic oscillator and od a charged particle in a timedependent electromagnetic filed. J. Math. Phys A, 1969, 10, P. 1458.

[12] Chen X., Ruschhaupt A., et al. Fast optimal frictionless atom cooling a harmonic traps: Shortcut to adiabaticity. Phys. Rev. Lett., 2010, 104, P. 063002.

[13] Torrontegui E., Ibanez M., et al. Shortcut to adiabaticity. Adv.At. Mol. Opt. Phys., 2013, 62, P. 117.

[14] Masuda S., Nakamura K. del Campo A. High-fidelity rapid ground-state loading of an ultracold gas into an optical lattice. Phys. Rev. Lett., 2014, 113, P. 063003.

[15] Torrontegui E., Martinez-Garaot M., Ruschhaupt A., Muga J.G. Shortcut to adiabaticity: fast-forward approach. Phys. Rev. A, 2012, 86, P. 013601.

[16] Takahashi K. Fast-forward scaling in a finite-dimensional Hilbert space Phys. Rev. A, 2014, 89, P. 042113.

[17] Kato T. On the Adiabatic Theorem of Quantum Mechanics. J. Phys. Soc. Jpn., 1950, 5, P. 435.

[18] Messiah A. Quantum Mechanics 2. North-Holland, Amsterdam, 1962.

[19] Berry M.V. Quantal Phase Factors Accompanying Adiabatic Changes. Proc. R. Soc. London, 1984, 392, P. 45.

[20] Aharonov Y., Anandan J. Phase change during a cyclic quantum evolution. Phys. Rev. Lett., 1987, 58, P. 1593.

[21] Berry M.V. Geometric Amplitude Factors in Adiabatic Quantum Transitions. Proc. R. Soc. London Ser. A, 1990, 430, P. 405.

[22] Khujakulov A., Nakamura K. Scheme for accelerating quantum tunneling dynamics. Phys. Rev. A, 2016, 93, P. 022101.

[23] Nakamura K., Khujakulov A., et al. Fast forward of adiabatic control of tunneling states. Preprint submitted for publication. 